Apidologie, 1976, 7 (2), 169-187.

\title{
NOTES SUR LA BIOLOGIE D'HERIADES TRUNCORUM L. (HYMENOPTERA MEGACHILIDAE)
}

\author{
Beitrag zur Biologie von Heriades truncorum $L$. \\ (Hymenoptera Megachilidae)

\begin{abstract}
Maria de Lourdes MACIEL DE ALMEIDA CORREIA * 86600 Lusignan
\end{abstract} \\ Laboratoire d'Éthologie et d'Écologie des Insectes, I.N.R.A.,
}

\section{SUMMARY}

\author{
NOTES ON THE LIFE HISTORY OF Heriades truncorum L. \\ (Hymenoptera Megachilidae)
}

A survey concerning the life history of Heriades truncorum L., a palaearctic solitary species, is given. It was trapped in bamboopipes set in a $30-40 \mathrm{~km}$ radius around Lusignan**. These wooden traps have 3 rows of holes in different diameters set with the bamboo pipes.

This device is well accepted by this species. The egg always lies on the front part of stores facing the nest entrance. The development at the constant temperature of $22{ }^{\circ} \mathrm{C}$ is carried out in this manner : length of egg incubation : 3-4 days; feeding on pollen : 15-16 days; cocoon spinning : 8-14 days; prenymphal stage : 94-124 days; white pupa stage : $3-12$ days; coloured-eyed pupa stage : $5-11$ days; black pupa stage : $5-8$ days; period between moulting of imago and emergence : $1-5$ days.

A short description of all stages is given. Four species of parasites were found in these nests (genus Anthrax, Melittobia, Trichodes and Stelis).

\section{RESUMÉ}

Nous présentons ici un aperçu concernant la biologie d'Heriades truncorum L., espèce paléarctique solitaire. Nous l'avons fait nidifier dans des nichoirs à tubes de bambou placés dans un rayon de 30 à $40 \mathrm{~km}$ de Lusignan. Ces nichoirs en bois possèdent trois rangées de trous de diamètres différents, occupés par des tubes de bambou.

* Boursière de la Fondation Calouste Gulbenkian.

** Town located in the Middle West of France. 
Cette espèce accepte bien ce dispositif. L'cuf est toujours disposé sur la partie antérieure des provisions vers la sortie du nid. Le développement à température constante de $22{ }^{\circ} \mathrm{C}$ s'effectue de la manière suivante : durée d'incubation de l'ceuf : 3 à 4 jours: alimentation sur pollen : 15 à 16 jour; ; filage du cocon : 8 à 14 jours; stade prénymphal : 94 à 124 jours; stade nymphe blanche : 3 à 12 jours; stade nymphe yeux pigmentés : 5 à 11 jours; stade nymphe noire : 5 à 8 jours; période entre mue imaginale et émergence : 1 à 5 jours.

Une brève description de tous les stades est faite. Quatre espèces de parasites ont été obtenues dans ces nids (genres Anthrax, Melittobia, Trirhodes et Stelis).

\section{INTRODUCTION}

L'information bibliographique sur le genre Heriades est très réduite. LABoulbene en 1873 publie une brève note sur la nidification de l'espèce paléarctique Heriades truncorum L. et son parasite Anthrax aethiops Fab. Malyshev en 1936 publie une note sur l'approvisionnement des cellules par les abeilles solitaires et présente le schéma d'un nid d'Heriades truncorum L. Michener en 1938 publie un travail sur la systématique des abeilles américaines appartenant au genre Heriades. FISCHER en 1955 donne une description de nid d'Heriades variolosus Cres. Matrhews en 1965 décrit la biologie d'Heriades carinata Cres. Michener en 1968 publie une note sur la nidification d'Heriades spiniscutis Cam.

En France Heriades truncorum L. a été surtout étudié sur le plan de la systématique par Benoist (1929). Nous avons donc abordé l'étude de sa biologie en cherchant à apporter des précisions sur son cycle de développement, la structure de son nid et son comportement de construction. Toutes les étapes du développement et la durée de chacune ont été également observées, ainsi que les parasites les plus fréquents.

Notre étude est loin d'être complète, mais elle enrichit les connaissances sur la famille des Megachilidae.

\section{MATÉRIEL ET MÉTHODES}

\section{Sites de piégeage}

En se basant sur les données des années précédentes, nous avons installé en 1974 un total de 113 nichoirs dans sept endroits différents aux alentours de la Station d'Amélioration des Plantes Fourragères de Lusignan '. Ces emplacements sont indiqués sur la figure I. Une huitième localité (Barbezieux) à $150 \mathrm{~km}$ de Lusignan a été choisie en raison de l'abondance d'espèces solitaires.

Le système de piégeage a été celui déjà employé par TAser (1972). Envisageant une analyse approfondie pour les années à venir nous avons disposé à chaque endroit le même nombre de nichoirs orientés de la même façon. L'influence de l'orientation serait à tester dans nos études futures. Dans les localités suivantes : Barbezieux, Chêne, Mongadon, Pétinière et Verrines, on a disposé des groupes de quatre nichoirs à des hauteurs différentes : $0,50 \mathrm{~m} ; 1 \mathrm{~m}$; $1,50 \mathrm{~m} ; 2 \mathrm{~m}$ à partir du sol. (Planche $\mathrm{I}, \mathrm{a}$.) Le diamètre des tubes de bambou proposés variait de $2,50 \mathrm{~mm}$ à $5 \mathrm{~mm}$ et leur longueur de $5,2 \mathrm{~cm}$ à $6,5 \mathrm{~cm}$ respectivement.

1. La Station d'Amélioration des Plantes Fourragères se situe dans le Centre Ouest de la France, dans le département de la Vienne. 


\section{Observation au laboratoire}

Après avoir ramené au lahoratoire les nids complets des nichoirs (une fois par semaine), nous les observons de façon à noter tous les détails.

En retirant le lien élastique qui permet de garder en place les deux parties des tubes de bambou, nous passons à l'observation sous la loupe binoculaire. On note le nombre de cellules, la couleur des provisions et des bouchons, le mode de construction des nids. Ensuite nous les plaçons en étuve à température constante de $22^{\circ} \mathrm{C}$. Deux fois par semaine nous procédons à l'observation de ces nids et à la notation des étapes du développement.

Mensurations des bouchons initiaux, bouchons terminaux, cloisons, provisions et longueurs des cellules

Ces mesures sont effectuées à l'aide d'un micromètre oculaire sous la loupe binoculaire.

\section{RÉSULTATS}

\section{L'Adulte}

Les Heriades sont des abeilles solitaires de petite taille possédant un dimorphisme sexuel net et faisant leur apparition dès juin. Deux espèces sont rencontrées dans la région de Lusignan. Heriades truncorum L. et Heriades crenulatus Nyl. nidifiant toutes les deux dans des bambous de petit diamètre et bouchant leurs nids avec une substance résineuse de couleur et consistance variables, durcissant avec le temps. Notre étude concerne la seule espèce Heriades truncorum L., plus abondante que Heriades crenulatus.

La femelle a environ 6-8 mm de long (planche $1, \mathrm{~b}$ ); le mâle, 5-7 mm, est complètement noir sauf à la partie postérieure des tergites abdominaux où des poils blancs sont visibles.

Les mâles plus petits que les femelles sont facilement reconnaissables à l'absence de brosse sternale, à la plus grande densité de poils jaunâtres sur le front, les joues et le clypeus, et à la présence de fossettes sur le dernier sternite abdominal. Le $6^{\mathrm{e}}$ tergite abdominal est creusé de deux dépressions contiguës. Les femelles possèdent quatre séries de poils jaunes (brosse ventrale) sur les derniers sternites abdominaux. Cette brosse sert à ramasser le pollen. La partie antérieure du clypeus presque glabre possède deux petits tubercules rapprochés.

\section{Activité saisonnière}

Heriades truncorum L. est une espèce monovoltine, active unc partie de l'année, de juin à octobre (M. Correia sous presse). Le maximum d'activité est atteint en août (fig. 2). D'après les données obtenues en 1973 et 1974 nous avons pu déterminer l'abondance selon les lieux (tabl. 1), ainsi que le pourcentage de nidifications mensuelles pendant les deux années (fig. 3). 
Nb. nids

construits


FıG. 2. - Activité de nidification mensuelle d'Heriades truncorum L. dans la région de Lusignan au cours des années 1970 à 1974.

Axe des $x$ : mois de nidification.

Axe des y : nombre de nids construits.

Aвв. 2. - Nestbautätigkeit von Heriades während eines Monats in der Gegend von Lusignan in den Jahren 1970-1974.

Abszisse $=$ Nestbau-Monate.

Ordinate $=$ Anzahl gebauter Nester 




Fig. 3. - Occupation des nichoirs par Heriades truncorum L. en fonction de la période de l'année.

Verticalement : Pourcentage Nombre de nids construits / Nombre de tubes proposés. Horizontalement : mois de nidification.

Aвв. 3. - Besetzen der Nistanlagen durch Heriades in Abhängigheit von der Jahreszeit. Ordinate : Prozentsatz Zahl der gebauten Nester / Zahl der vorgeschlagenen Röhren. Alsszisse : Nestbau-Monate.

Une observation plus attentive des nidifications au cours des deux années aux différents emplacements de piégeage (tabl. 1) nous permet de tirer quelques conclusions : les insectes sont abondants dans certains lieux (Jazeneuil et Grand-Breuil) et rares en d'autres (Barbezieux).

\section{Activité de nidification}

La nidification dans des nids artificiels à tubes de bambou est importante, variant selon les années et la période de vol (Correia sous presse).

L'insecte construit en moyenne 4 à 6 cellules par nid disposées linéairement et séparées par des cloisons très fines. En présence de tubes de diamètre variant entre $2,5 \mathrm{~mm}$ à $5,0 \mathrm{~mm}$, il montre une préférence bien nette pour ceux de $3,0 \mathrm{~mm}$ (fig. 4). La longueur moyenne des cellules varie de $7,8 \mathrm{~mm}$ à $9,0 \mathrm{~mm}$ selon le diamètre choisi (tabl. 2). 
TавL. 1. - Abondance de nids d'Heriades truncorum L. par localité. Lusignan 1973-74.

TAB. 1. - Vorkommen v. Heriades-Nestern nach Standort, Lusignan 1973/74.

\begin{tabular}{|c|c|c|c|c|c|c|}
\hline \multirow[b]{2}{*}{$\begin{array}{l}\text { Localités } \\
\text { Standorte }\end{array}$} & \multicolumn{3}{|c|}{1973} & \multicolumn{3}{|c|}{1974} \\
\hline & \begin{tabular}{|} 
Nombre de \\
Nids \\
Zahl d. \\
Nester
\end{tabular} & $\begin{array}{l}\% \text { de tubes } \\
\text { occupés } \\
\% \text { beset } /- \\
\text { ter Röbren }\end{array}$ & $\begin{array}{c}\text { Nombre de } \\
\text { cellules } \\
\text { par nid }\end{array}$ & $\begin{array}{c}\text { Nombre de } \\
\text { Nids } \\
\text { ZahL d. } \\
\text { Nester }\end{array}$ & $\begin{array}{l}\% \text { de tubes } \\
\text { occupés } \\
\% \text { besetz- } \\
\text { ter Röhren }\end{array}$ & $\begin{array}{c}\text { Nombre de } \\
\text { cellules } \\
\text { par nid } \\
* *\end{array}$ \\
\hline Barbezieux $\ldots \ldots \ldots \ldots$ & 4 & 0,9 & 4,2 & 2 & 0,7 & 3 \\
\hline Servolet $\ldots \ldots \ldots \ldots \ldots$ & 0 & 1 & 1 & 6 & 5 & 2 \\
\hline Chene $\ldots \ldots \ldots \ldots \ldots$ & 2 & 0,9 & 3,0 & 5 & 2 & 2 \\
\hline Jazeneuil $\ldots \ldots \ldots \ldots \ldots$ & 13 & 22 & 3,0 & 6 & 20 & 2 \\
\hline Mongadon $\ldots \ldots \ldots \ldots \ldots$ & 1 & 0,7 & 3,0 & 10 & 6 & 3 \\
\hline Petinière $\ldots .$. & 8 & 7 & 4 & 3 & 0,8 & 2,0 \\
\hline Verrines $\ldots \ldots \ldots \ldots \ldots$ & 8 & 4 & 4 & 1 & 0,2 & 4,0 \\
\hline Grand-Breuil ........... & 6 & 13 & 3 & 0 & / & l \\
\hline $\begin{array}{l}\text { Moyenne générale } \ldots \ldots \ldots \\
\text { Durchsehnitt } \\
\text { llgemein }\end{array}$ &  & 3,29 & 3,26 & & 1,94 & 2,33 \\
\hline
\end{tabular}

* et ${ }^{* *}$ Anzahl Zellen je Nest.



FIo. 4.--Choix du diamètre du tube de nidification par les femelles.

Axe des $x$ : diamètre en $\mathbf{m m}$.

Axe des y : nombre de nids.

Авв. 4. - Auswahl durch die Weibchen nach Röhrendurchmesser.

Abszisse $=$ Durchmesser in $\mathbf{m m}$.

Ordinate $=$ Anzahl der Nester. 


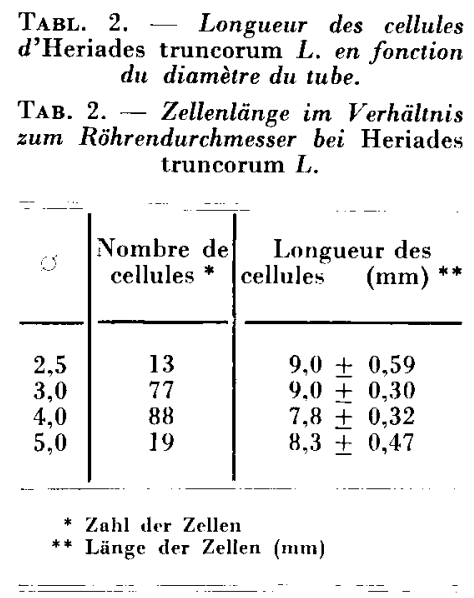

Les bouchons sont en substance de consistance résineuse, de texture, odeur et couleur variables, durcissant avec le temps. Bien souvent des particules végétales sont incluses dans la résine rendant meilleure la consistance des bouchons. Une analyse chimique de cette substance est envisagée. L'épaisseur du bouchon initial varie de $1,9 \mathrm{~mm}$ à $2,3 \mathrm{~mm}$, celle du bouchon terminal de $2,7 \mathrm{~mm}$ à $3,3 \mathrm{~mm}$. La couleur de la substance résineuse varie du jaune clair, transparent au jaune foncé presque marron. Deux nids ayant des bouchons et des cloisons de couleur verdâtre ont été trouvés dans plusieurs sites. La femelle tapisse légèrement, d'une substance analogue à celle des bouchons, toute la paroi interne des tubes de bambou. Celle-ci serait selon Fischer (1955) mélangée à des secrétions salivaires. Lorsque le bouchon initial est terminé, la femelle passe à l'approvisionnement de la cellule. Les deux tiers de chaque cellule sont remplis de pollen jaune ou blanc provenant de plantes appartenant à la famille des Composées (les genres Senecio, Lappa, Cirsium, Crepis, Inula ont pu être identifiés).

Un seul cuf est déposé par cellule dans une légère dépression du centre de la provision. Ensuite la femelle cloisonne la cellule à l'aide d'une substance identique à celle des bouchons et passe à la cellule suivante. Les cloisons sont minces (entre $1,0 \mathrm{~mm}$ à $1,2 \mathrm{~mm}$ ). Entre la dernière cellule et le bouchon terminal, elle laisse un compartiment d'environ deux fois la longueur de la cellule et qui selon Fischer (1955) servirait à l'aération. La durée de construction du nid varie selon les mois de l'année, donc selon la température et très certainement selon l'âge de la femelle. Les périodes de construction et le nombre de nids sont donnés par la figure 5 . 




F1G. 5. - Evolution de l'activité de nidification sur quatre mois pendant les années 1973/1974. Axe des y : nombre de nids construits.

Aвв. 5. - Entwicklung der Nestbautätigkeit im Verlauf von vier Monaten in den Jahren 1973/74. Ordinate = Zahl der gebauten Nester.

\section{Développement larvaire}

\section{L'ouf}

L'cuf de 1,6-1,7 mm de longueur, est allongé, blanc, transparent, à chorion lisse et brillant. Il est normalement déposé dans un creux de la provision, verticalement par rapport à celle-ci (planche 11, a); sa partie antérieure est dirigée vers la sortie du tube. Au fur et à mesure que l'embryon se développe, nous remarquons une zone plus foncée vers la base. Cette zone va devenir centrale formant une masse jaune au milieu de l'œuf, les extrémités étant blanches et transparentes.

La période d'incubation à température constante de $22^{\circ} \mathrm{C}$ varie de 3 à 4 jours. Le nombre d'œufs pondus en 1973 est de 99 pour un effectif de 137 cellules construites (38 cellules sont approvisionnées sans auf). Pendant l'année 1974 le nombre d'œufs est de 77 pour 77 cellules terminées. 
La larve et le flage du cocon

La larve est de type apode. Elle commence à se nourrir dès l'éclosion. La différenciation de la tête et la segmentation de la jeune larve est bien évidente. Le passage aux différents stades larvaires est assez difficile à observer. Nous avons essayé de marquer nos larves de premier stade en déposant très délicatement des particules de terre très fine. Cette méthode n'a permis aucun résultat car la poussière provoquait le dessèchement des larves. Une méthode plus appropriée est donc à mettre au point pour déterminer le nombre de stades larvaires.

La durée du développement larvaire varie de 39 à 55 jours. La larve se nourrit jusqu'à la construction du cocon. La majorité des larves après avoir consommé leur nourriture commence à déféquer, mais nous en avons trouvé quelques-unes qui commençaient à rejeter leurs déjections avant d'avoir terminé leurs provisions. Les excréments sont généralement allongés, rougeâtres ou d'un jaune très clair selon les couleurs des provisions.

Lorsque la larve âgée arrête de se nourrir, elle commence le filage du cocon. Les déjections sont rassemblées et disposées sur le côté droit de chaque cellule. Puis à l'aide d'une sécrétion salivaire elle tapisse toute la paroi de la cellule donnant ainsi une sorte de protection au cocon (planche 11, b). Le filage du cocon dure de 9 à 14 jours. Le cocon est fin, opaque et soyeux. Il est formé d'une couche transparente de fils de soie. Sur les bords antérieur et postérieur les fils sont plus visibles. Nous avons également remarqué dans quelques cellules, une sorte de capuchon filé sur le côté apical du cocon et le recouvrant légèrement. Fischer (1955) note déjà la présence de ce capuchon sur le côté interne des cloisons chez Heriades variolosus, et MatтHEWs (1965) remarque une sorte d'opercule chez Heriades carinata. TAYLoR (1962) signale une structure similaire dans les nids d'Heriades freygressneri.

La diapause se passe à l'état de prénymphe et nous avons remarqué, sur un lot de cinq nids conservés en étuve à température constante de $22{ }^{\circ} \mathrm{C}$, que la nymphose se produisait au bout de 94 à 125 jours. Quelques jours avant l'émergence la nymphe toute blanche commence à noircir, d'abord au niveau des yeux et des ocelles, puis du thorax, de l'abdomen, des antennes, jusqu'à devenir complètement noire. (planche 11 , fig. $c$ et $d$ ).

Bien que dans certains nids, les adultes émergent le même jour, d'autres nids sont caractérisés par une émergence échelonnée. Ce mode est plus fréquent et semble provenir de l'échelonnement dans le temps de la construction des différentes cellules.

\section{Sex-ratio}

Le nombre d'adultes sortis en 1973 est de 12 femelles et 30 mâles pour l'espèce Heriades truncorum L. et 7 femelles et 6 mâles pour Heriades crenulatus Nyl. 


\section{Parasitisme}

Le taux de parasitisme en 1973 a été de $21,2 \%$ sur les 137 cellules construites et en 1974 de $11,7 \%$ sur les 77 cellules achevées (tabl. 3). Il est réparti de la façon suivante : 7,3\% dus à Stelis pour l'année 1973 et 9,1\% pour 1974. Parasités par Anthrax 2,9\% pour 1973 et par Trichodes 1,5\% en 1973 et $1,3 \%$ en 1974 .

TABL. 3. - Abondance des ennemis trouvés dans les nids d'Heriades truncorum $L$. au cours des années 1973 et 1974.

ТАв. 3. - Zahl der in den Jahren $1973 / 74$ in den Heriades-Nestern gefundenen Feinde.

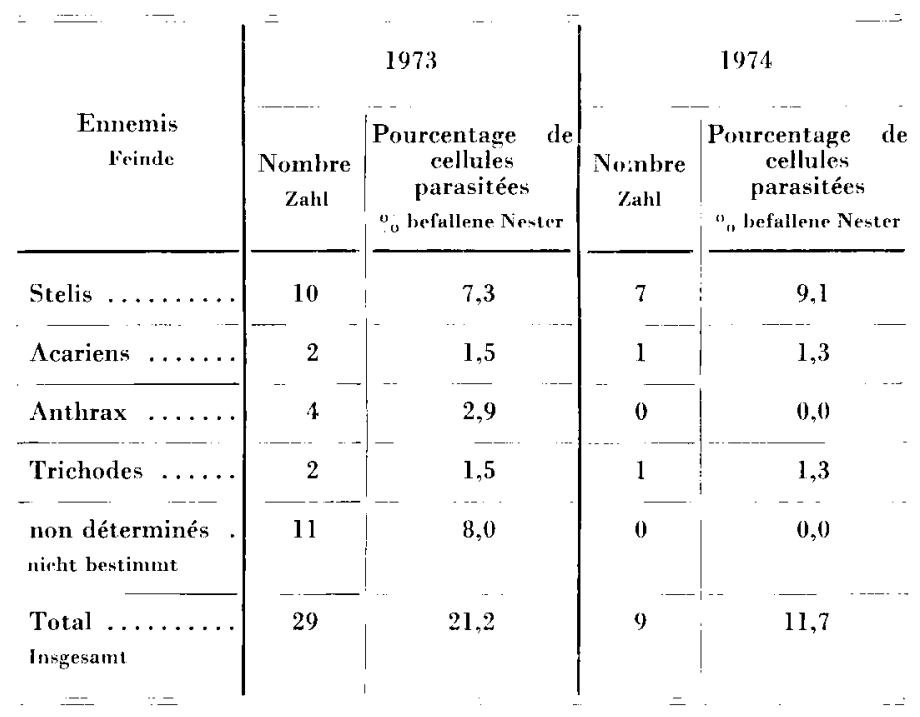

\section{DISCUSSION}

Passant en revue les étapes les plus importantes du cycle de développement d'Heriades truncorum L., nous remarquons que l'œuf a en moyenne $1,6 \mathrm{~mm}$ de long et que l'incubation dure entre 3 et 4 jours. L'hibernation se passe à l'état de prénymphe. Par contre $H$. freygessneri Sch. passe l'hiver à l'état de nymphe (TAYLOR 1962). L'ouf d'Heriades carinata (Michener 1968) a environ $2-2,3 \mathrm{~mm}$ de long et l'incubation dure 5 jours $1 / 2$. La défécation commence 6 jours après l'éclosion et la construction du cocon 25 jours après l'éclosion. La durée de construction du cocon est de 7 jours une fois la nourriture achevée. La période larvaire dure 30 jours et pendant la deuxième semaine d'août dans l'état du Michigan la dernière larve d'Heriades carinata est au stade prénymphal. 
Heriades truncorum L. construit son nid en disposant ses cellules de façon linéaire. Les bouchons et les cloisons sont bien distincts et la substance qui les constitue reste à analyser. Il nous semble qu'elle est obtenue à partir d'espèces végétales existant à proximité des sites de nidification. Chez Heriades spiniscutis dont l'émergence des adultes est décalée par rapport à Heriades truncorum (Michener 1968) le nid est construit dans du bois mort et les cloisons ne sont pas toujours présentes. La source de " résine " employée dans la construction du nid est le latex d'Euphorbia angularis.

Pour Matthews (1965) la source de résine demeure inconnue, mais il doute qu'elle ait son origine dans le sumac. Dans quelques nids d'Heriades truncorum L. nous avons remarqué la présence de petits morceaux de végétaux mélangés à la substance résineuse. Chez Heriades variolosus Fischer (1955) signale que du sable ou de petits cailloux s'y trouvent inclus.

La période de nidification d'Heriades truncorum L. se situe entre juin et octobre avec un pic de sorties en août. L'analyse pollinique montre une grande spécificité de butinage pour les Composées (Senecio, Lappa, Cirsium, Crepis, Inula). Pour Heriades carinata Cress. (Matrhews 1965) la période de nidification varie de mi-juin à mi-juillet et atteint un pic le 30 juin au Michigan. Dans l'Orégon cette même espèce apparaît plus tard et plus longtemps (11 juillet) avec un pic fin juillet présentant des fluctuations jusqu'à complète disparition à la fin d'août. Cette espèce récolte du pollen sur une gamme importante de fleurs appartenant à plusieurs familles : Asclepias syriaca, Monarda fistulosa, Melilotus alba, Rhus glabra, Cichorium intybus, Apocynum et surtout Rhus sp. (sumac).

Quant au taux de parasitisme il est assez élevé : $21,2 \%$ en 1973 et $11,7 \%$ en 1974. Matthews (1965) trouve un pourcentage de $1,2 \%$ des cellules occupées par Stelis vernalis Mitchell (Megachilidae) et $0,4 \%$ par Sapyga louisi Krombein (Sapygidae).

\section{CONCLUSION}

Au cours de cette étude nous avons souligné l'importance de la méthode de piégeage, du diamètre des tubes proposés, des sites de nidification.

Le monovoltisme de l'espèce Heriades truncorum L. ainsi que les périodes de vol (de juin à octobre avec un maximum de sorties en août) sont mis en évidence.

L'élevage en étuve à température constante de $22^{\circ} \mathrm{C}$ nous a permis de suivre les différents stades du développement jusqu'à l'émergence. La durée moyenne de l'éclosion est de 4 jours et celle de la période d'alimentation de 16 jours. La durée de filage du cocon est de 11 jours et le stade prénymphe 
dure en moyenne 124 jours. La durée moyenne du stade nymphe blanche est de 11 jours, et le noircissement complet obtenu 16 jours plus tard. 3 à 4 jours s'écoulent entre la mue imaginale et l'émergence de l'adulte.

La nidification étant plus abondante dans certains lieux par rapport à d'autres indique que les facteurs d'abondance tels que le microclimat, la végétation, le type de sols, restent à analyser.

D'autre part la variation mensuelle et annuelle des nidifications est certainement dûe aux facteurs climatiques tels que la température, le degré d'humidité, la pluviométrie, la vitesse du vent, l'exposition au soleil et l'orientation.

Dans une note ultérieure, nous essayerons de déterminer le degré d'importance de ces facteurs climatiques.

Reçu pour publication en janvier 1976.

Eingegangen im Januar 1976.

\section{REMERCIEMENTS}

Nous remercions M. LeconTE pour ses conseils au cours de ce travail, M. TASEI pour l'aide apportée au long de cette étude et pour la détermination des pollens.

Nous tenons également à remercier M. Desmier de Chenon (I.N.R.A.) pour l'identification des Heriades, ainsi que M. CARRE pour la documentation photographique de notre étude.

\section{ZUSAMMENFASSUNG}

Heriades truncorum L., eine palaearktische, solitäre, monovoltine Art, die in der Gegend von Lusignan * häufig vorkommt, war Gegenstand unserer Untersuchungen. Die Aufzuchten wurden nach der Methode von TASEI (1972) durchgeführt. An sieben verschiedenen Plätzen in der Umgebung der Station d'Amélioration des Plantes Fourragères haben wir insgesamt 113 Nistgelegenheiten aufgestellt. Unsere Arbeit umfasste Beobachtungen im Freien und im Labor.

Unter den besonderen Bedingungen der Gegend von Lusignan haut Heriades ihre Nester in der Zeit von Juni bis Oktober, wobei die Hauptaktivität in den Monat August fällt.

Die Verteilung der Nester, die je nach Art der Fallenanlagen verschieden ist, weist auch Unterschiede nach den Jahren auf.

Heriades sammelt mit Vorliebe auf Kompositen (Inula, Senecio, Cirsium, Pieris) und ihr zahlenmässig starkes Auftreten ist an solchen Orten, wo sowohl die klimatischen Bedingungen als auch die vorhandene Flora günstig sind, besonders auffällig.

Eine kurze biologische Untersuchung brachte genauere Kenntnis über ihren Entwicklungszyklus. Das 1,6-1,7 mm grosse Ei ist länglich und wird normalerweise senkrecht in eine kleine, mit Vorräten versehene Grube abgelegt. Bei einer konstanten Temperatur von $22{ }^{\circ} \mathrm{C}$ beträgt die Inkubationszeit 3-4 Tage, die Zeit der Futteraufnahme 16 Tage. Das Stadium des Kokonspinnens dauert 8-14. Tage, während das Stadium der Pronymphe 94-124 Tage umfassen kann.

* Stadt im Mittelwesten Frankreichs, 28 km von Poitiers entfernt. 
3-12 Tage dauert das Stadium der weissen Made, 5-11 Tage das der Made mit ausgefärbten Augen. Das Stadium der schwarzen Nymphe dauert 5-8 Tage.

Der Bau des Nestes, die Art seiner Anlage und die Entwicklungsstufen des Insekts im Verlauf des Jahres werden beschrieben. Vier Arten von Parasiten wurden in diesen Nestern festgestellt (Anthrax, Melittobia, Trichodes und Stelis).

\section{RÉFÉRENCES BIBLIOGRAPHIQUES}

Benoist R., 1929, Les Heriades de la faune française. (Hymenoptera Apidae.) Ann. Soc. Ent. Fr. $98: 131-141$.

Correra M., Fluctuations de populations naturelles chez les genres Chelostoma et Heriades (Hymenoptera Megachilidae) (sous presse).

Fischer R. L., 1955, A nest of Heriades variolosus (Cress) (Hymenoptera Megachilidae) Canad. Ent. $87: 33-36$.

Laboulbene A., 1873, Note sur la nidification de l'Heriades truncorum et sur l'Anthrax aethiops, parasite de cet Hyménoptère. Ann. Soc. Ent. de France, 42 : 57-60.

Malyshev S. I., 1936, The nesting habits of solitary bees. A comparative study. Eos 11 : 201309.

Matthews R. W., 1965, The biology of Heriades carinata Cresson (Hymenoptera Megachilidae.) Contrib. Amer. Ent. Inst. 1 (3): 1-33.

Mrchener C. D., 1938, American bees of the genus Heriades. Ann. Ent. Soc. Amer. 31 : $514-531$.

Michener C. D., 1968, Heriades spiniscutis, a bee that facultatively omits partitions between rearing cells (Hymenoptera, Apoidea.) Journ. Kans. Ent. Soc. $41: 484-493$.

Stephen W. P., Bohart G. E., Torchio P. F., 1969, The biology and external morphology of bees. With a synopsis of the genera of Northwestern America. Oregon Agr. Expt. Stat. $1-140$.

Taser J.-N., 1972, Observations préliminaires sur la biologie d'Osmia coerulescens L., pollinisateur de la luzerne. Apidologie 3 (2) 149-165.

TAser J.-N., 1973, Le comportement de nidification chez Osmia (Osmia) cornuta Lat. et Osmia (Osmia) rufa L. (Hymenoptera Megachilidae.) Apidologie 4 (3) 195-225.

TAYLon J. S., 1962, Notes on Heriades freygessneri Schletterer (Iymenoptera Megachilidae.) Journ. Ent. Soc. S. Africa 25 : 133-139. 
Planches

\section{TAFEL}




\section{PLANCHE I}

Localité de piégeage Adulte d'Heriades truncorum $L$.

a. - Le Chêne : disposition des nichoirs à des hauteurs différentes.

b. - Femelle d'Heriades truncorum $L$.

1. brosse ventrale

2. tubercules de clypeus.

$1 \mathrm{~mm}$

\section{TAFEL I}

Fangstellen von adulten Heriades truncorum $L$.

a. - Eiche : Nistanlagen in verschiedenen Höhen

b. - Heriades - Weibchen

1. Bauchbürste

2. Clypeus-Hörnchen

$1-1 \mathrm{~mm}$ 

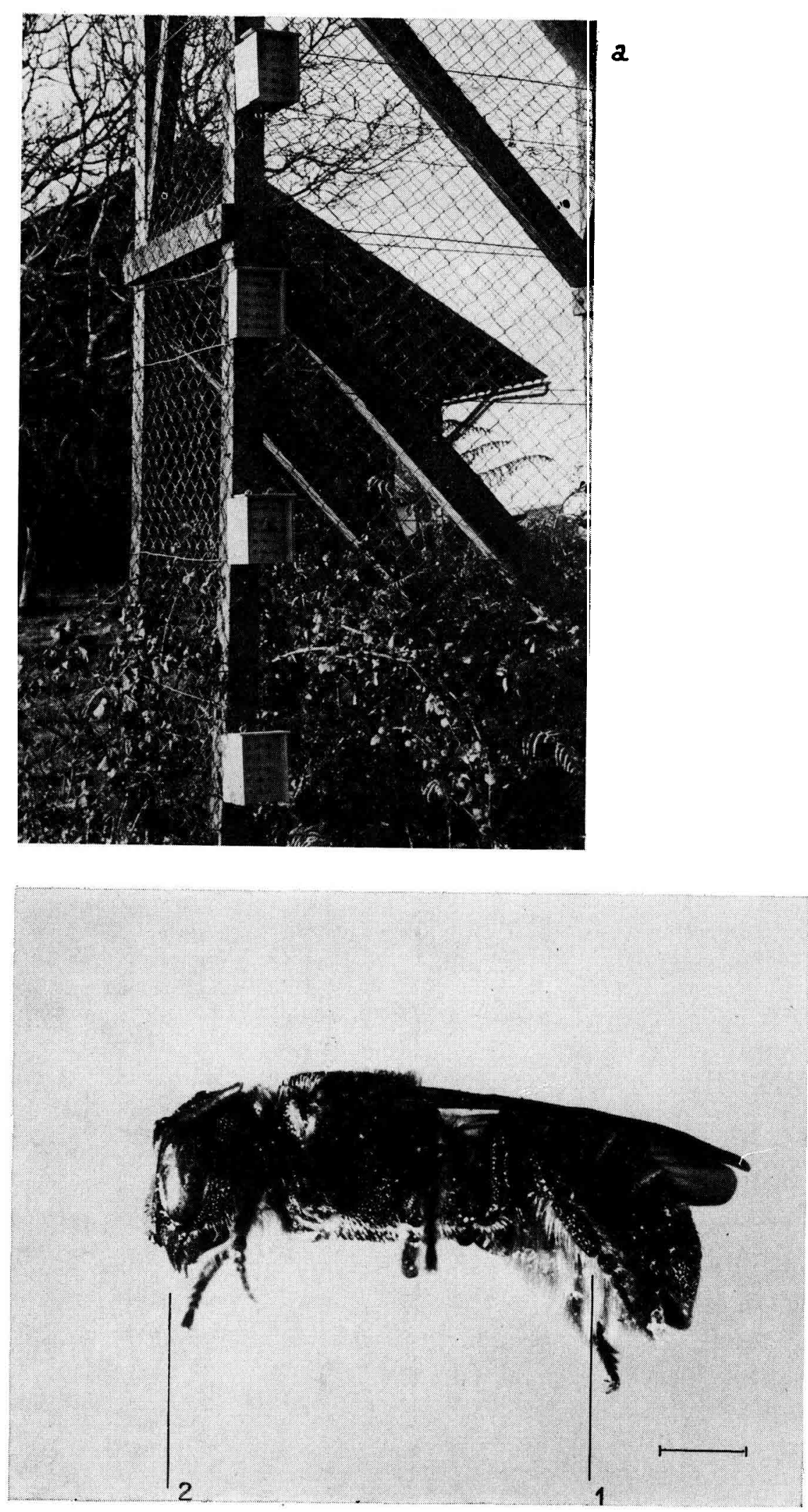


\section{PLANCHE II}

\section{Stades de développement d'Heriades truncorum $L$.}

a. - Nid; vue d'ensemble.

1. provisions

2. cloison

3. ouf

4. bouchon initial

b. - Dêtail du nid.

1. cloison

2. excréments

3. cocon c. - Étapes du développement. 1. larve de premier stade

2. larve de dernier stade.

3. nymphe blanche

4. nymphe yeux pigmentés

5. nymphe noire

d. - Détail d'un nid dans une paille en carton.

1. bouchon initial

2. larve de dernier stade

3. nymphe blanche aux yeux rouges

4. nymphe blanche aux yeux noirs

TAFEL II

Entwicklungsstadien von Heriades truncorum $L$.

a. - Nest, Gesamtansicht.

1. Vorräte

2. Scheidewand

3. Ei

4. Anfangspfropfen

b. - Einzelheiten des Nestes.

1. Scheidewand

2. Exkremente

3. Kokon c. - Entwicklungsstufen.

1. Made im ersten Stadium

2. Made im letzten Stadium

3. weisse Nymphe

4. Nymphe, Augen gefärbt

5. schwarze Nymphe

d. - Einzelheiten eines Nestes in einem Strohhalm in einem Karton.

1. Anfangspfropfen

2. Made im letzten Stadium

3. weisse Nymphe mit roten Augen

4. weisse Nymphe mit schwarzen Augen 



$\boldsymbol{\sigma}$

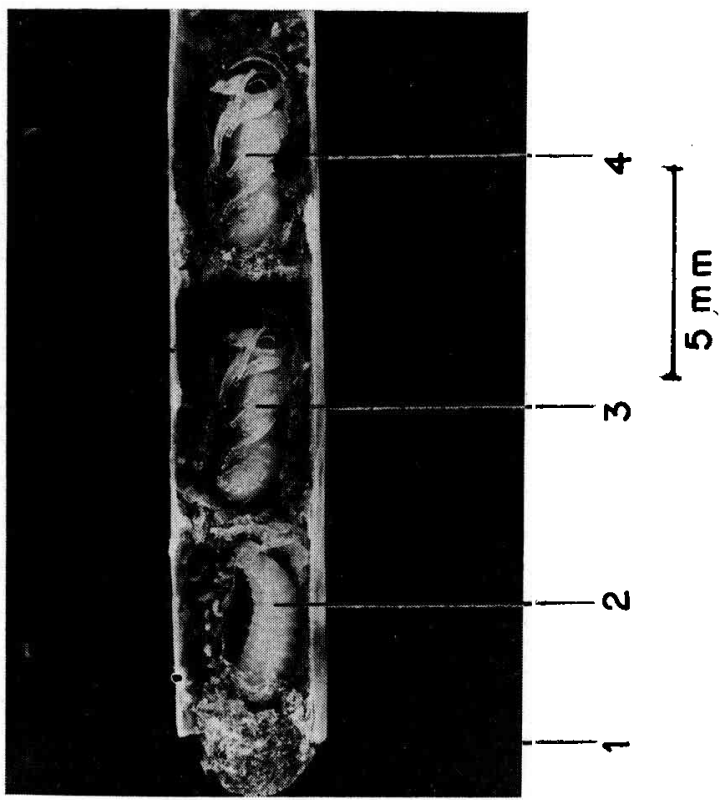

